\title{
Implementing a Proactive Deprescribing Approach to Prevent Adverse Drug Events
}

\author{
Timothy S. Anderson, MD, MAS ${ }^{7}$ (D), Parag Goyal, MD, MSC ${ }^{2}$, and Zachary A. Marcum, \\ PharmD, $P h D^{3}$ \\ 'Division of General Medicine, Beth Israel Deaconess Medical Center Boston, MA, USA; ${ }^{2}$ Divisions of Cardiology and General Internal Medicine, \\ Weill Cornell Medicine New York, NY, USA; ${ }^{3}$ University of Washington, School of Pharmacy Seattle, WA, USA.
}

$J$ Gen Intern Med 35(12):3694-6

DOI: $10.1007 / \mathrm{s} 11606-020-05886-\mathrm{z}$

(c) Society of General Internal Medicine 2020

\begin{abstract}
A dverse drug events (ADEs) are a leading cause of preventable harm in the USA. The majority of ADEs are experienced by older adults and are most commonly related to medications taken for chronic conditions (e.g., anticoagulants, hypoglycemics, and analgesics). ${ }^{1}$ After an ADE occurs, the complicit medication is usually discontinued - a process we term reactive deprescribing. While necessary, reactive deprescribing is not sufficient to optimize patient safety and wellbeing. To move beyond reacting to ADEs and instead prevent them, widespread adoption of proactive deprescribing, whereby thorough review and routine reconsideration of the risks and benefits of chronic medications are incorporated into clinical care, is needed (Table 1). Despite a growing evidence base including observational studies ${ }^{2}$ and randomized trials ${ }^{3}$ increasing physician awareness of deprescribing, implementation of proactive deprescribing in the US healthcare system faces structural challenges related to reimbursement, time, and communication. We believe that overcoming these challenges can be achieved by building upon existing Medicare programs to provide a venue to implement proactive deprescribing in a reimbursable and sustainable fashion.
\end{abstract}

\section{BARRIERS TO PROACTIVE DEPRESCRIBING}

The benefits and risks of chronic medications shift over time due to changes in an individual's health status, cognition, function, and goals. Certain classes of chronic medications are frequently inappropriate for older adults (e.g., anticholinergic and sedative/hypnotic drugs), while the appropriateness of other medications depends on the individual context of each patient. For example, a patient newly diagnosed with Parkinson's disease faces increased disease-related risks of

Received February 6, 2020

Accepted April 28, 2020

Published online May 14, 2020 falls and mood disorders. As a result, the additive risks posed by previously tolerated antihistamines (taken for seasonal allergies) and beta-blockers (taken for hypertension) may now outweigh their benefits. Ideally, the prescribing physician would discuss proactively deprescribing these medications. However, several health system barriers make this challenging. ${ }^{4}$ First, due to a fragmented system, the prescriber may not be aware of the patient's new diagnosis for months. Second, the time needed to discuss the risks and benefits of chronic medications during an office visit often competes with acute problems and other healthcare maintenance. Third, clinicians may be hesitant to change medications prescribed by others and may face additional barriers to effectively communicating with other clinicians regarding medication management. Each of these factors contributes to prescribing inertia and constitutes a barrier to proactive deprescribing. ${ }^{5}$ When inertia prevails up until an $\mathrm{ADE}$ is experienced, the opportunity for prevention is lost.

\section{IMPLEMENTING PROACTIVE DEPRESCRIBING THROUGH EXISTING MEDICARE PROGRAMS}

To counteract prescribing inertia, structured programs facilitating the adoption of proactive deprescribing into routine clinical practice are essential. A successful proactive approach will require deprescribing discussions at regularly scheduled intervals and following major health events (e.g., hospitalizations). Key components of deprescribing protocols have been developed and include reconciliation of a patients' medications and their indications, discussion of medication risks and benefits, shared-decision making discussions on stopping medications with the lowest ratio of benefits to harms, and close monitoring following medication changes. ${ }^{4}$ These steps require substantial time with the patient and in pre-visit planning. Focused efforts are also needed to enhance communication between primary and specialty clinicians to develop cohesive patient-centered recommendations. Two existing Medicare programs could potentially facilitate the implementation of proactive deprescribing: annually, as a part of Medication Therapy Management (MTM) programs, and following hospitalizations, as part of Transitional Care Management (TCM) programs. 
MTM programs are a required part of Medicare prescription drug plans and are offered to beneficiaries meeting minimum criteria including multiple chronic conditions, multiple medications, and a likelihood of exceeding prespecified medication cost thresholds. The components of MTM programs closely mirror the necessary steps for deprescribing and include an annual person-to-person or telehealth comprehensive medication review, quarterly targeted medication reviews, and interventions targeting both beneficiaries and prescribers. Prior studies suggest MTM programs may improve medication appropriateness and reduce ADEs, but less than half of recommendations are accepted by prescribers. ${ }^{6}$ Many MTM programs deliver recommendations through faxed or mailed letters which may be overlooked by prescribers, whereas partnered models involving pharmacist and physician teams have shown more promise.

As Medicare continues to innovate and improve upon the current MTM program structure, consideration should be given to specifying the inclusion of proactive deprescribing discussions. MTM deprescribing initiatives could involve pharmacists identifying potential prescribing cascades (e.g., prescriptions for medications to treat the side effect of another medication) and prescriptions for preventative medications that are unlikely to benefit patients with limited life expectancy (e.g., statin prescription for patient with $<6$-month prognosis due to cancer), among others. As part of the development of an enhanced MTM innovation model, a technical expert report highlighted the need for pharmacist consultation as a top priority for improving MTM program impact and reducing ADEs. ${ }^{5}$ Enhanced pharmacist roles within MTM programs would be well-suited for implementing deprescribing given that pharmacists have the necessary skills to provide expertise on medication risks across disease states and to facilitate communication between primary and specialty care providers (see Table 1 for example). In fact, some of the most promising empirical evidence to-date on the benefits of deprescribing high-risk medications in older adults comes from pharmacist-led interventions. ${ }^{3}$

The second promising avenue for implementing proactive deprescribing is Medicare's TCM program. Billing for TCM codes provides increased reimbursement for ambulatory visits following hospitalization in an effort to improve postdischarge outcomes. The post-hospitalization period is an especially appropriate time to engage in proactive deprescribing, given its significance with regard to changes in health status and disease trajectory, and also the known increased risks of ADEs following hospitalization. Hospitalizations often result in multiple medication changes, new diagnoses, and changes in healthcare goals. Each of these events may influence the risks and benefits of other chronic medications, which may be challenging to address during the hospital stay where acute issues are prioritized and the physicians caring for the patient may have limited knowledge of the patient's goals or chronic disease control.

TCM visits offer an opportunity to consider proactive deprescribing at important periods of a patient's health

Table 1 Comparison of Reactive and Proactive Deprescribing

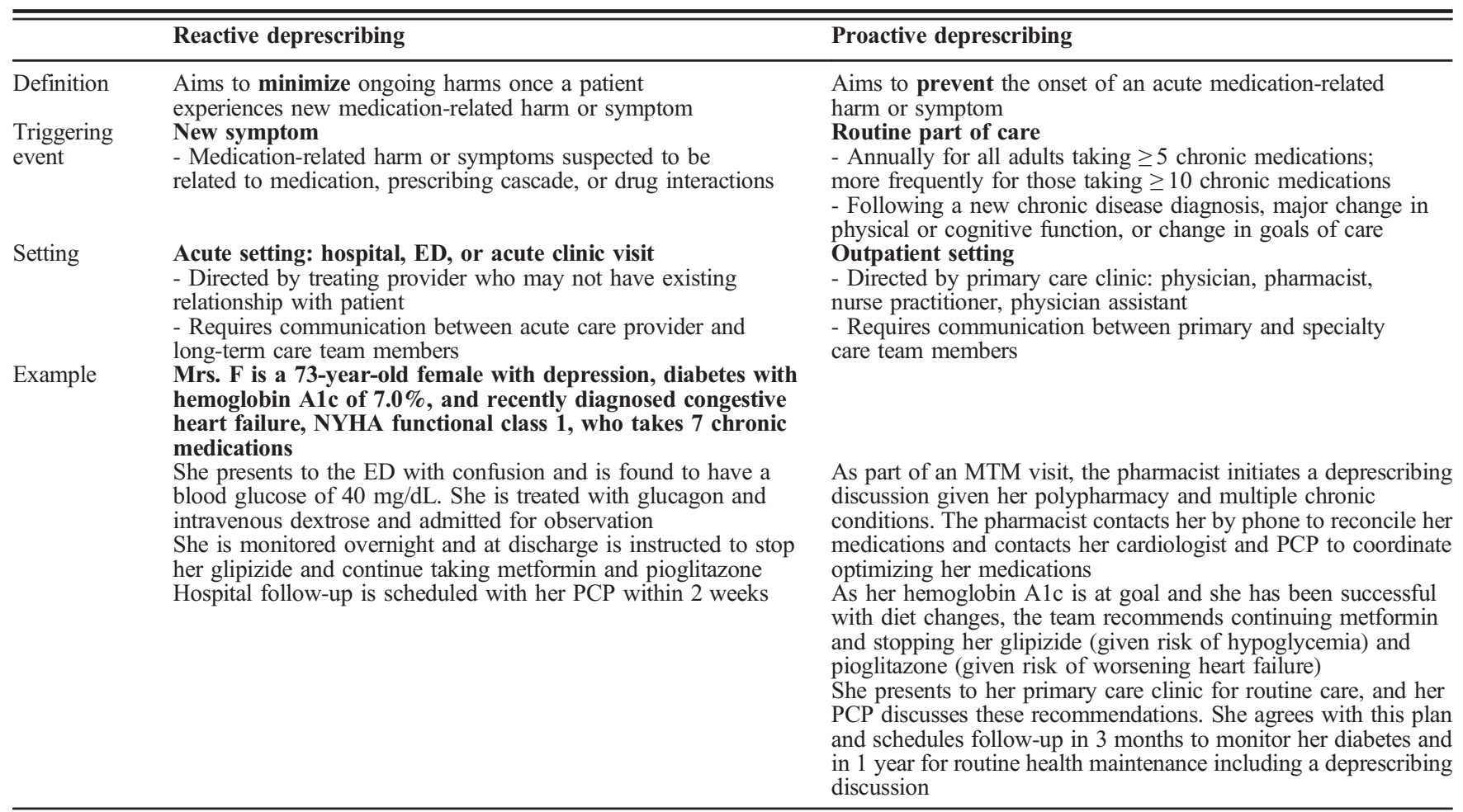


trajectory but require careful coordination. A TCM visit might identify opportunities to proactively deprescribe duplicate therapies mistakenly taken by patients, medications prescribed at discharge posing severe drug interactions, or prehospitalization medications which may no longer benefit the patient after a new diagnosis. Recent research indicates use of TCM services reduces mortality and decreases costs, yet TCM services remain underutilized, possibly in part because TCM reimbursement requires both communication with the patient within 2 days and an office visit within 7 to 14 days after discharge. ${ }^{7}$ One way to enhance utilization of TCM services is through enhanced roles for pharmacists. A growing evidence base supports collaborative pharmacist prescribing models; however, laws governing pharmacist provider status (ability to bill Medicare for services) vary greatly between states and are not yet recognized nationally. Pharmacists are currently unable to bill for TCM services, yet, similar to MTM programs, with forward-thinking policy changes, pharmacists could play a key role in coordinating TCM services and leading proactive deprescribing efforts following hospitalization.

\section{CONCLUSION}

To reduce medication-related harms in older adults, we must work to emphasize and prioritize proactive deprescribing. Existing Medicare programs provide a starting point for overcoming prescribing inertia and adopting deprescribing into routine practice. To fully realize the promise of deprescribing to prevent adverse drug events, continued development of policies and systems are needed to incentivize these novel approaches.

Acknowledgments: We thank Michael Steinman, MD (University of California, San Francisco) for review of prior drafts of this manuscript.
Corresponding Author: Timothy S. Anderson, MD, MAS; Division of General Medicine, Beth Israel Deaconess Medical Center Boston, MA, USA (e-mail: tsander1@bidmc.harvard.edu).

Funding Information Dr. Anderson is supported by grants from the National Institute on Aging (L3OAG060493 and R03AG064373). Dr. Goyal is supported by grants from the National Institute on Aging (R03AG056446) and the American Heart Association (18IPA34170185). Dr. Marcum is supported by the National Institute on Aging (K76AG059929).

\section{Compliance with Ethical Standards:}

Conflict of Interest: The authors declare that they do not have a conflict of interest.

Disclaimer: The contents represent the views of the authors only and not necessarily those of Beth Israel Deaconess Medical Center, University of Washington, Cornell Medicine, or the National Institutes of Health.

\section{REFERENCES}

1. Shehab N, Lovegrove MC, Geller AI, Rose KO, Weidle NJ, Budnitz DS. US Emergency Department Visits for Outpatient Adverse Drug Events, 2013-2014. JAMA. 2016;316(20):2115-2125.

2. Scott IA, Hilmer SN, Reeve E, et al. Reducing inappropriate polypharmacy: the process of deprescribing. JAMA Intern Med 2015; 175:827-834.

3. Martin P, Tamblyn R, Benedetti A, Ahmed S, Tannenbaum C. Effect of a Pharmacist-Led Educational Intervention on Inappropriate Medication Prescriptions in Older Adults: The D-PRESCRIBE Randomized Clinical Trial. JAMA. 2018;320(18):1889-1898.

4. Anderson K, Stowasser D, Freeman C, Scott I. Prescriber barriers and enablers to minimising potentially inappropriate medications in adults: a systematic review and thematic synthesis. BMJ Open. 2014;4(12):e006544.

5. Steinman MA, Landefeld CS. Overcoming Inertia to Improve Medication Use and Deprescribing. JAMA. 2018;320(18):1867-1869.

6. Centers for Medicare \& Medicaid Services. Part D Enhanced Medication Therapy Management Model. 2019. https://innovation.cms.gov/initiatives/enhancedmtm/. Accessed January 7, 2020.

7. Bindman AB, Cox DF. Changes in health care costs and mortality associated with transitional care management services after a discharge among Medicare beneficiaries. JAMA Intern Med 2018;178:1165-1171.

Publisher's Note: Springer Nature remains neutral with regard to jurisdictional claims in published maps and institutional affiliations. 